\title{
An Observational Study on Infectious Etiology of Hypo and Hyper Pigmented Macular Lesions in the Skin in a Tertiary Care Hospital
}

\author{
Rizwanasheik, J. Manonmoney ${ }^{*}$ and K. V. Leela
}

SRM Medical College Hospital and Research Centre, SRM Institute of Science and
Technology, Kattankulathur - 603203, Kancheepuram District, Tamilnadu, India

*Corresponding author

\section{A B S T R A C T}

\begin{tabular}{|l|}
\hline Ke y w o r d s \\
Hypopigmentation, \\
Hyper \\
pigmentation, \\
Macular lesions, \\
Malassezia, \\
Dermatophyte test \\
medium (DTM)
\end{tabular}

\begin{abstract}
Melanin pigment is a complex, brown - black polymer. Excessive pigmentation is known as hyper pigmentation and decreased pigmentation is known as hypopigmentation. Genetic and severe environmental conditions can cause melanin disruption. Dermatophytic infections were characterized by hypo pigmentation and hyper pigmentation macules. The present study aims to identify the infectious aetiology of hypo pigmented and hyper pigmented macular lesions in the skin other than Leprosy using conventional techniques among patients attending a tertiary care hospital. This is an observational study approved by Institutional ethical committee Skin scrapings were collected under strict aseptic precautions as per standard protocol. Potassium hydroxide preparation-KOH mount made and observed under microscopy. The $\mathrm{KOH}$ positive samples are cultured in Dermatophyte Test Medium and Sabourauds Dextrose Agar. A total of 120 samples were collected, out of which $56(46 \%)$ were $\mathrm{KOH}$ positive and 64(54\%) were negative In this study the overall prevalence rate of dermatophytic infection was highest among males 35(64\%) and in females $19(35 \%)$. It is important toidentify the causes and treat them appropriately to reduce the psychosocial stress of patients with pigmentary disorder.
\end{abstract}

\section{Introduction}

Melanin pigment is a complex, brownpolymer, synthesized from dihydroxphenylalanine by DOPA oxidiase and tryosinase in organelles known as melanosomes. These organelles are injected via dendrites into keratinocytes. (1) Genetic and severe environmental conditions can cause melanin disruption. It's important to identify the root cause before undergoing treatment. Altered skin pigmentation can result from increased or decreased melanin, abnormal melanin distribution, decreased hemoglobin, or deposition of exogenous substances

Excessive pigmentation is known as hyper pigmentation and decreased pigmentation is known as hypo pigmentation. Both may be localized or generalized, non-melanin pigments may be also cause skin darkening(1). The scale become more prominent on rubbing or scraping the lesions, it is mostly caused by the fungi of malassezia species. Pityriasis alba disorder mainly affects children, it may be present as 
hypo to de pigmented macula, it also response to vitiligo in determine leprosy, post inflammatory hypo pigmentation and hypo pigmentation polymorphic light eruption (2).

Other localized areas of hyper pigmentation may occur after skin inflammation, a more diffuse type of facial hyper pigmentation that is mostly seen in women is known as chloasma (2).

The treatment for this hyper and hypo pigmentation depends upon the type of disease they are several common and un common hypo pigmentation disorders, it is important to know the psychological stress of the patients caused by pigmentation disorders (2).

Malasseziosis is a fungal infection caused by various species of genus malassezia, These species are usually considered as resident flora of man and animals. some species are responsible for variety of superficial as well as systemic infections, Pityriasis versicolor being the most commonly presenting diseases. The other conditions in which this lipophilic fungus plays important etiological role are seborrheic dermatitis, folliculate and allied illnesses (2). Based on molecular biology of malassezia, most of the controversies prevailing for the last so many years have resolved to a great entailing new interest in its clinical importance. The lipophilic yeast like fungus with its natural habits in stratum corneum of human skin as resident flora causes Pityriasis versicolor and has been implicated in pathogenesis of many dermatomes (2).

The objective of the study was to identify infectious etiology of hypo and hyper pigmentation macular lesions in the skin other than leprosy using conventional techniques in patients attending a tertiary care hospital in kancheepuram district (South India), in a period of about twelve months, from January
2019 to January 2020.

\section{Materials and Methods}

It was an observational study conducted in 120 patients who are dealing with superficial fungal skin infection with hypo or hyper pigmentation. Institutional Ethics Committee has authorized this study (1584/IEC/2019 on 27.2.2019). The study group included clinically suspected hypo and hyper pigmentation macular lesions in skin in all age groups of both males and females other than leprosy. Patients with subcutaneous and deep skinlesions and leprosy are excluded. skin scrapings obtained from the patients were subjected to microscopy and culture as per protocol (2). Patient information sheet consent form as been obtained duly signed by the participated in this study.

\section{Sample collection}

\section{Specimen collection: (2)}

Skin Scrapings: Grossly contaminated skin or that to which antifungal agents have been applied skin was decontaminated with $70 \%$ alcohol and skin scales were scraped off from the margins using blunt scalpel on a black paper. Invading hyphae tend to grow radially from the centre of the lesion which become devoid of viable fragments as acquired local immunity develops.

\section{Samples transport and storage}

The keratinous materials (Skin) samples were dried out to prevent the overgrowth of saprophytic bacteria and fungi.

Proper care was taken that there was no moisture in the clinical specimens prior to processing.

A black paper was folded to form a pocket and an envelope was designed for the sample collection. 
The collected clinical samples were transported appropriately and processed.

The Specimen should not be refrigerated as the viability of some species of dermatophyte may be affected.

\section{Conventional techniques}

\section{Direct microscopy - potassium hydroxide (KOH) mount (2)}

Potassium hydroxide $(\mathrm{KOH})$ solution was prepared and used for the direct detection of fungal elements in samples. Because $\mathrm{KOH}$ which is an alkaline solution used to clear the cell debris and to observe fungal element in a wet mount preparation. $10 \%, 40 \% \mathrm{KOH}$ were used for skin scraping, hair and nail specimens respectively. The collected samples were initially subjected to direct microscopy under $10 \mathrm{X}$ and high power $40 \mathrm{X}$ objective to observe the fungal elements and their morphology.

\section{Procedure}

The little amount of clinical sample is transferred on to the glass slide containing $\mathrm{KOH}$.

The prepared slides were placed within a Petri dish containing moistened cotton to prevent from drying and to provide the adequate moisture.

The prepared slide was allowed, for the $\mathrm{KOH}$ to act on the clinical specimen to provide digestion of the keratinous material for half an hour of incubation and if required incubation was extended further overnight.

The specimen digested by $\mathrm{KOH}$ was observed under low power (10X) objective and the entire area under the cover slip was scanned from end to end in a zigzag manner.
If any fungal elements were suspected, it was examined under high power (40X) objective. Presence of branching, type of branching, the color, septation and thickness of hyphae were observed and noted.

\section{Culture}

Samples are collected from the suspected cases were cultured, which shows $\mathrm{KOH}$ mount (microscopy) positive.

The samples were inoculated on two different culture media like Sabouraud Dextrose Agar (SDA) with and without antibiotics and Dermatophyte test medium (DTM).

The inoculated culture media were incubated at three different temperatures like room temperature, $37^{\circ} \mathrm{c}$ and $25^{\circ} \mathrm{c}$ for 7 days to 21 days.

Rate of growth and growth characteristics were observed. Proper care was taken to avoid contamination (or else it may interfere with the susceptibility testing).

\section{Sabouraud dextrose agar (SDA) (2)}

Sabouraud dextrose agar (SDA) is the selective medium for the isolation of dermatophytes other fungi and yeast. The acidic $\mathrm{pH}$ of this medium (pH5.0) inhibits the growth of bacteria. Antibiotics like chloramphenicol-0.04 mg, gentamicin-5 mg, and cycloheximide- $0.50 \mathrm{mg}$ are added to inhibit bacterial growth.

\section{Procedure}

The samples were stabbed into the medium with a sterile inoculating loop.

The plates were incubated at room temperature in an inverted position. 
Culture plates were examined daily for growth during the first week. Plates with no growth were re-incubated and observed for 46 weeks before being reported as culture negative.

After the sufficient incubation temperature and conditions, the plates with confluent growth in the area of inoculation appeared a cottony, powdery and velvety with pigment on reverse. The pure growth of fungus was processed further for identification up to the species level as per standard algorithms.

\section{Dermatophyte test medium (DTM) (2)}

Dermatophyte test medium is a selective and differential medium used for the detection and identification of dermatophytes. On this medium the identification of dermatophytes were based on the morphology and the production of alkaline metabolites. A combination of three antimicrobial agents (cycloheximide, chlortetracycline and gentamicin) inhibits bacteria and saprophytic yeasts and moulds.

\section{Procedure}

The samples were stabbed into the medium with a sterile inoculating loop.

The plates were incubated at room temperature in an inverted position with increased humidity.

Culture plates were examined daily for growth during the first week. Plates with no growth were re-incubated and observed for 46 weeks before being reported as culture negative.

\section{Result and interpretation}

After the sufficient incubation temperature and conditions, the plates with confluent growth in the area of inoculation was looked like Cottony, powdery and velvety growth was observed on the Dermatophyte test medium. The dermatophyte changed the colour of the medium from yellow to pinkred. The pure growth of fungus was processed further for identification up to the species level as per standard algorithms.

\section{Microscopic identification (2)}

\section{Scotch tape preparation}

\section{Procedure}

The adhesive side of the transparent tape was touched with surface of the colony.

A drop of lacto phenol cotton blue solution was added on the slide and the transparent tape was placed on the slide containing the LPCB solution.

Observed microscopically for the characteristic shape and arrangement of the spores

The hyphae, septum, conidial arrangement and exact morphology of the fungus were observed.

\section{Tease mount preparation (2)}

Lacto phenol cotton blue (LPCB) is a stain used for making semi-permanent microscopic preparation of fungi. Phenol used to kill any live organism. Lactic acid preserves the fungal structure and cotton blue stain is absorbed by the hyaline fungal structures make them more distinct and glycerol provides the moisture.

\section{Procedure}

A small drop of lacto phenol cotton blue (LPCB) was placed in the centre of a clean 
glass slide.

A small portion of fungus material was taken from the culture using a bacteriological needle or spades and teased apart in the drop of lacto phenol cotton blue stain using a teasing needle and the spade.

The preparation was covered with cover slip and examined microscopically. The slide was preserved by sealing the edges of the cover slip with DPX mount. The excess moisture around the cover slip was removed by blotting paper before sealing cover slip.

The LPCB slide preparation was examined under low power (10X) and high power (40X) objective of microscope.

Addition of $10 \%$ polyvinyl alcohol (PVA) preserved the specimen as a permanent stain for mounting.

The characteristic arrangement of spores and types of hyphae, septae and exact morphology of the fungus, especially the characteristics of macro conidia and micro conidia were observed which enabled the identification of the species.

\section{Slide culture technique (2)}

The slide culture technique was in the study, used to study the undisturbed morphology of the fungus especially conidia, conidiophores and hyphae. The slide culture was needed when the tease mount was inconclusive.

\section{Procedure}

A sterile glass slide was placed on the bent glass rod at the bottom of the Petri dish. A block of the Sabouraud Dextrose Agar/ Potato Dextrose Agar was placed on the slide. The fungal strain was inoculated at four slides of the agar block. The inoculated block was covered with sterile cover slip and incubated at $25^{\circ} \mathrm{C}$. The filter paper was moistened with a little volume of sterile distilled water to avoid drying of the agar. After the growth appeared a small drop of LPCB solution was taken on a microscopic slide.With the forceps, the cover slip was removed carefully from the agar block. The cover slip was carefully placed on the LPCB mount.The slide was observed under low power and high-power objective.

The mycelium which gets adhered to the glass surface revealed the characteristic appearance which would have been lost during the teasing process.

\section{Results and Discussion}

The present study entitle an observational study on infectious etiology of hypo and hyper pigmented macular lesions in the skin in a tertiary care hospital, includes 120 patients and 120 skin scrapings were collected during the time period of January- 2019 to January 2020.

In this study out of 120 patients $71(59.16 \%)$ patients are males and $49(40.83 \%)$ were females. And $\mathrm{KOH}$ positive patients are 56 $(46 \%)$ and $\mathrm{KOH}$ negative are $64(54 \%)$.

And also, the patients those who are having hyper pigmentation were $62(51.6 \%)$ and hypo pigmentation were 58 (48\%).

The superficial fungal infections seen in the present study are T. Corporis 15 (26.8\%), T. Mannum 10 (17.8\%), T. Peddris 12 (21.4\%), T. cruris $6(10.7 \%)$, Malassezia species 3(5.4\%), Trichophyton species 10(17.9). 
Fig Koh mount result

Fig.1 Out of 120 (n) samples $\mathrm{KOH}$ positive samples are 56(46\%), $\mathrm{KOH}$ negative are 64(54\%)

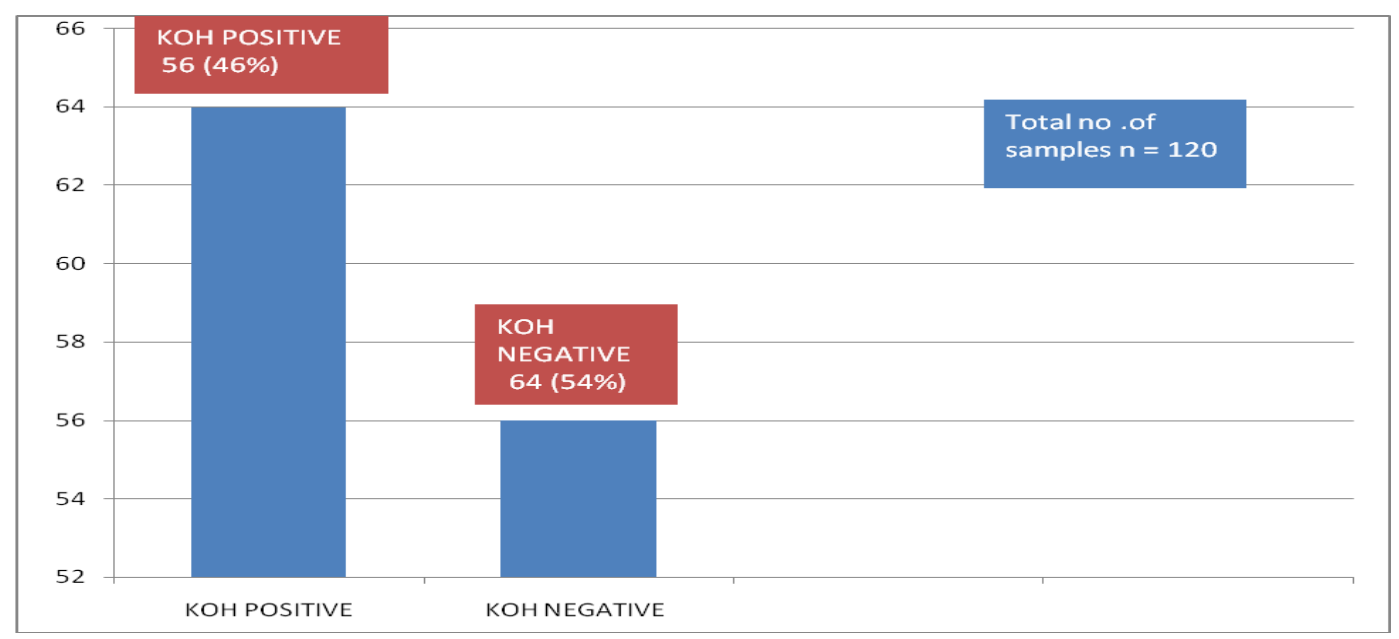

Fig.2 Total no. of samples $n=120$ males $71(59.16 \%)$ and females $49(40.83 \%)$

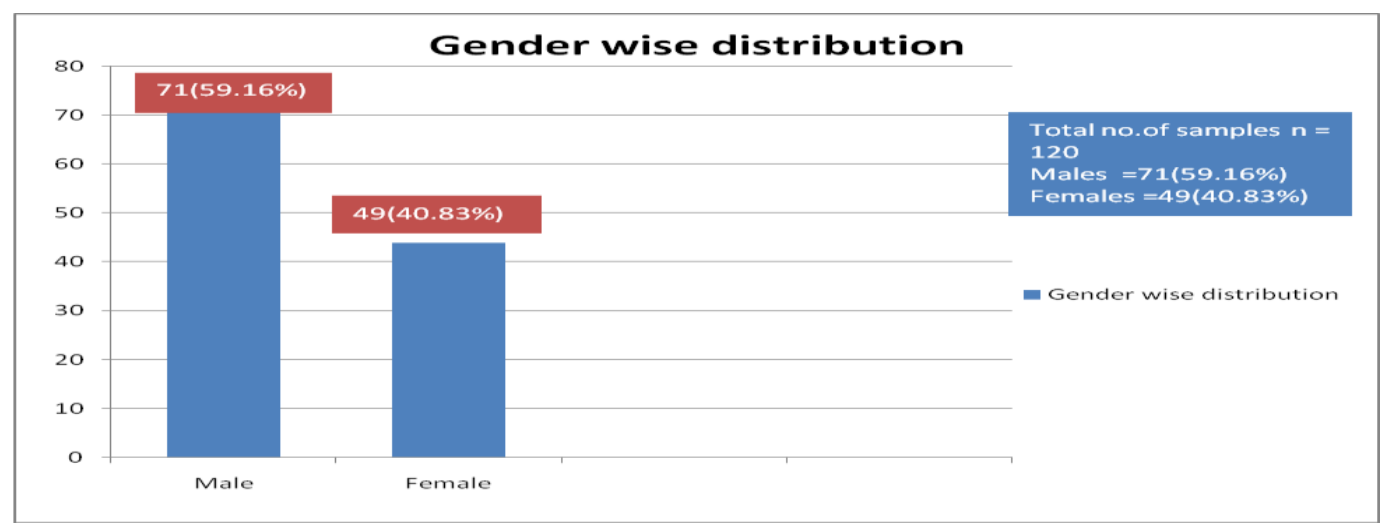

Fig.3 Out of 120 (n) samples hypopigmentation 62(51.6\%) and hyperpigmentation 58(48.3\%) Distribution of hypopigmented and hyperpigmented

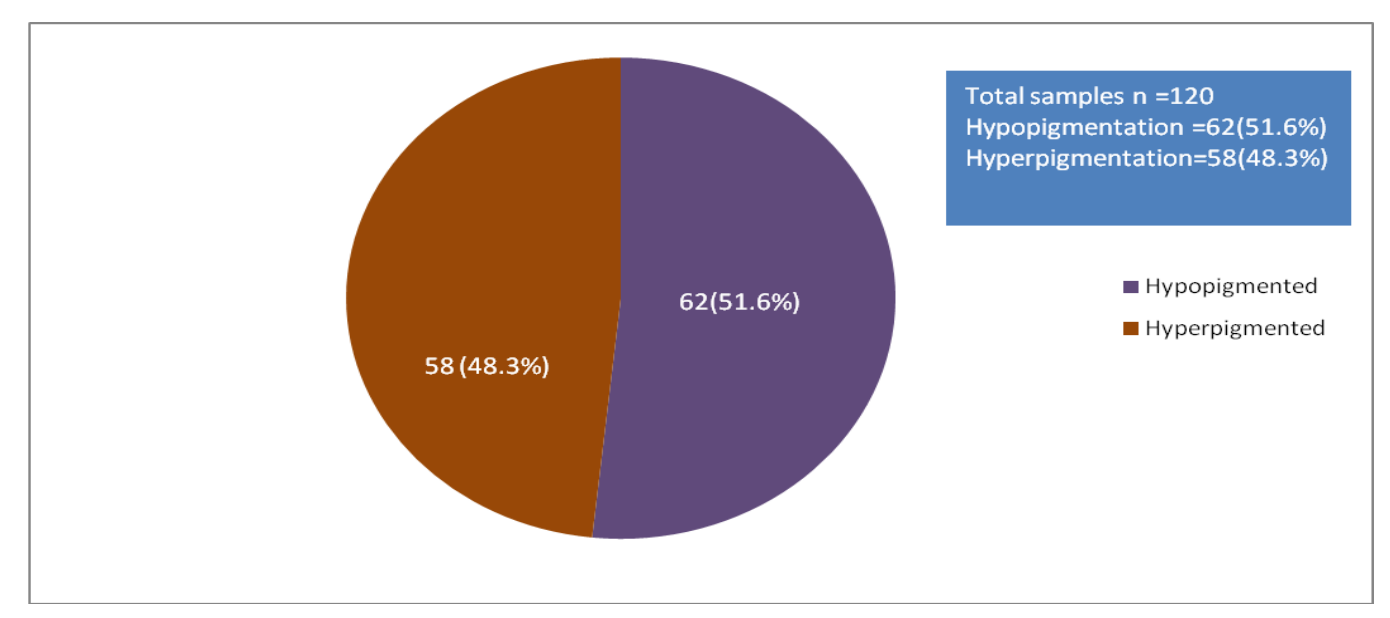


Fig.4 Out of 120 (n) sample superficial fungal clinical infection observed in patients

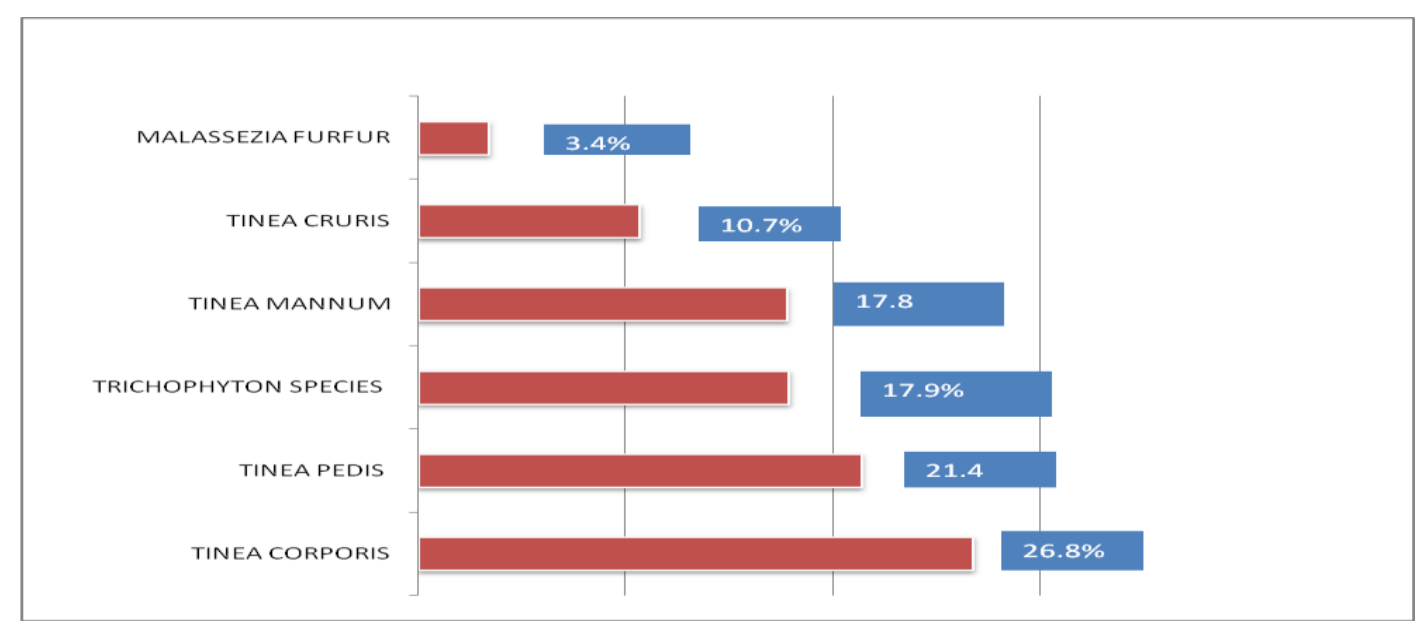

Fig.5 Methodology Algorithm

Skin scrapping sample (By proper protocol)<smiles>C=[Tl]</smiles>

Direct $\mathrm{KOH}$ Mount (To find fungal filaments or elements)<smiles>C=[Te]</smiles>

1. Saboraud's dextrose agar (SDA) with antibiotics

(chloramphenico 1-50.0mg and gentamicin-5.0mg)

2. SDA without antibiotic

3. Dermatophyte test medium (DTM)<smiles>[W]=[W]</smiles>

Incubation Temperature and Time

Incubate at $25^{\circ} \mathrm{c}, 30^{\circ} \mathrm{c}$ and $37^{\circ} \mathrm{c}$ “

. Rapid growth"

2. Moderately rapid growth

3. Slow growth<smiles>C=[Te]</smiles>

Slide Culture Method, Scotch Tape Method, Tease Mount Method

( For Morphological identification )(2). 
Image.1 Growth on dermatophyte test medium (dtm)

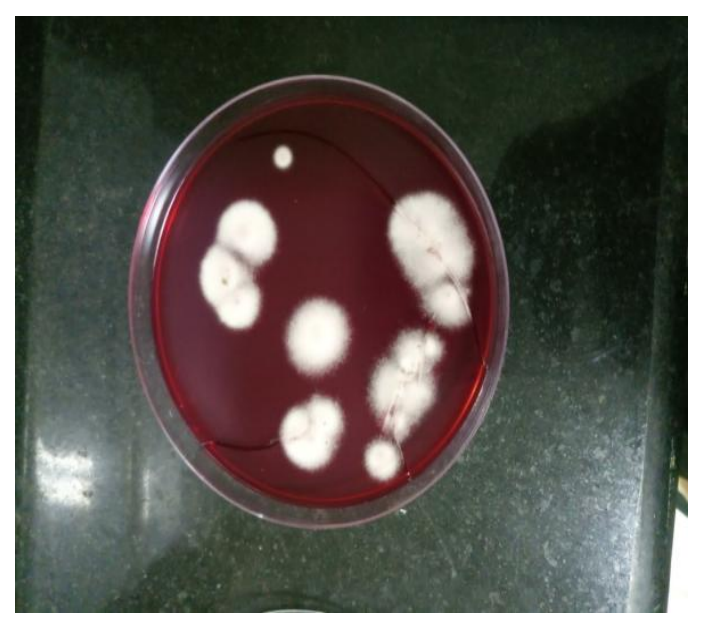

Image. 2 Growth on sda medium

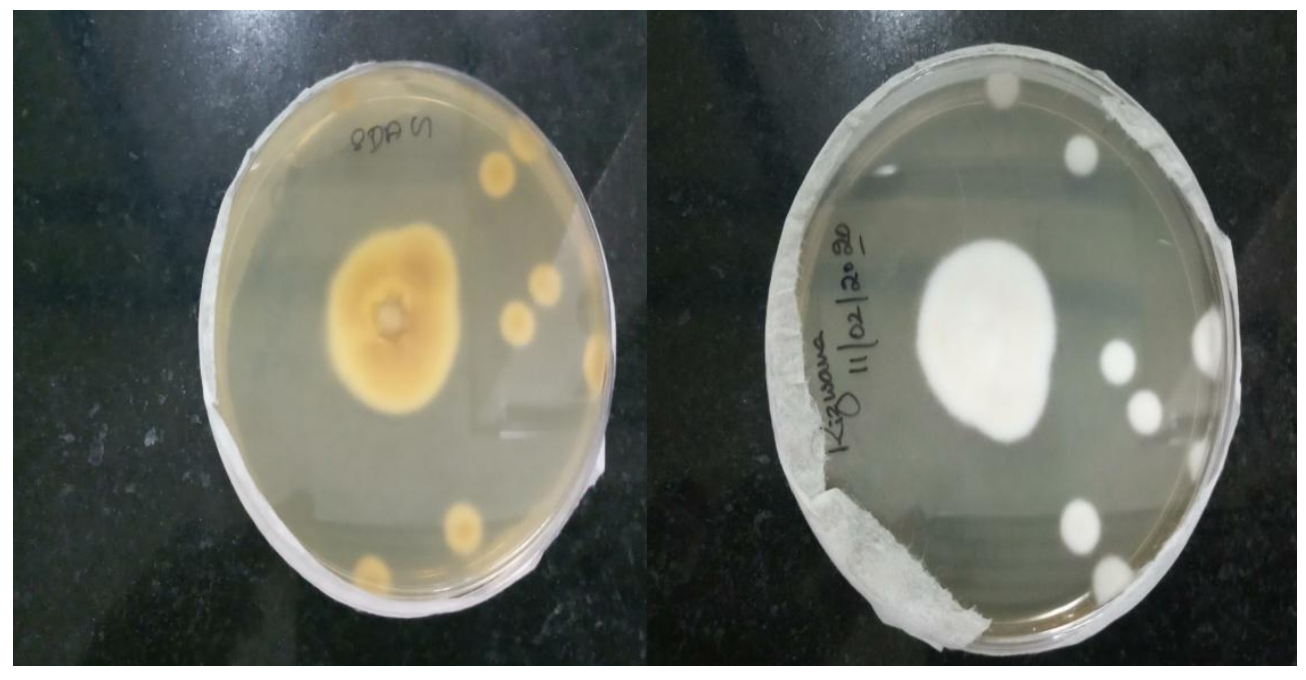

Image.3 LPCB slide for skin scrapping sample showing teardrop microconidia and few long pencil shaped macro condida

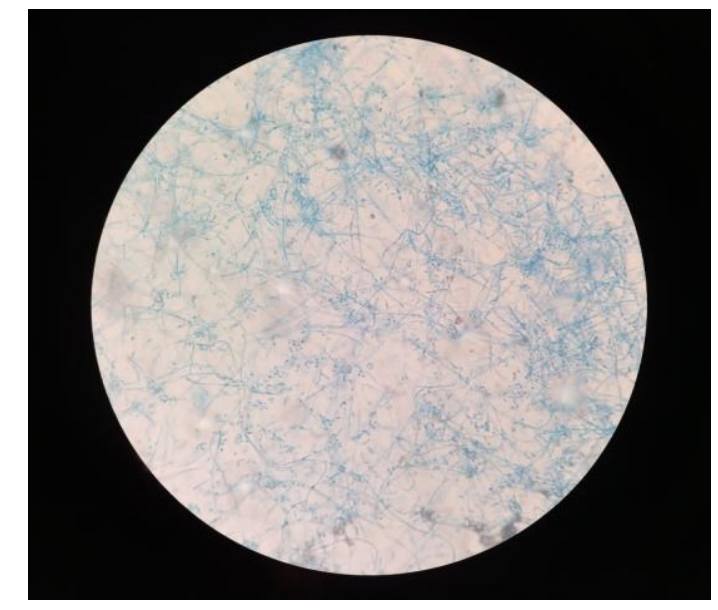


In Clinical mycological evaluation of dermatophytosis at tertiary care hospital in central India shows out of 150 patients Tinea corporis $53(35.5 \%)$ Tinea cruris $(22.6 \%)$ tinea barbae $(1.3 \%)$ 134(84.3\%) patients are positive by KOH mount 69 (46.0\%) culture.

In Distribution of Malassezia species study in patients with Pityriasis versicolor in kolar region observed that among 100 samples only 70 are $\mathrm{KOH}$ positive out of these $52(74.27 \%$ ) are hypo pigmented lesions and $18(25.17 \%)$ are hyper pigmented lesions.

Prevalence of different species of Malassezia causing Pityriasis versicolor and sites of distributions of lesions in a tertiary care hospital in these studies they founded that out of 108 cases $M$ furfur 61(56.8\%) M globosa30 (27.78\%) M.restricta109 (9.26\%) M. sympodialis 7 (6.48\%). The M. furfuris the most common superficial fungal infection observed.

They performed intermittent pulse dosed terbinafine in the treatment of Tinea corporis and Tinea cruris,and showed that taking intermittent pulsed terbinafine for time period of 3 weeks achieved a cure rate of $91.3 \%$ with a very low relapse rate in the treatment of Tinea corporis and Tinea cruris. The study describes the fungal infections in the skin, or dermatomycoses, are highly prevalent and may affect 20-25\% of the population worldwide and stated that superficial fungal infections comprise the majority of these cases, most commonly caused by dermatophytes, but Candida albicans and Malassezia are also common etiology agents.

In conclusion

In the present study patients those you are having clinical condition like hypo pigmentation were $62(51.6 \%)$ and hyper pigmentation were 58 (48\%).
Out of 120 patients $71(59.16 \%)$ patients are males and $49(40.83 \%)$ are females Out of the 120 patients $\mathrm{KOH}$ positive patients are 56 (46\%) and $\mathrm{KOH}$ negative are 64 (54\%). More number of dermatophytic infections found in males than female patients

Most commonly seen fungal infections with hypo and hyperpigmentation presentations are T. Corporis $15 \quad(26.8 \%)$, T. Mannum 10(17.8\%), T.pedis12(21.4\%) T. Cruris 6(10.7\%), Malassezia species 3 (5.4\%), and Trichophyton species 10(17.9\%).

\section{Acknowledgement}

I would like to offer my sincere thanks to Dr. K V Leela, M.D Professor and head of Microbiology for the support and unwavering guidance, tolerance. This would have not been possible without Dr. J Manonmoney Assistant Professor, Department of Microbiology.

\section{References}

1. Essential in dermatology by DM. Thappa 2 edition text book.

2. Jagdishchander Text book of Medical Mycology $4^{\text {th }}$ edition.

3. Distribution of Malassezia species in patients with pityriasis versicolor in kolarregion byBanu raju from Indian journal of medical microbiology may 2008, 53:182-5

4. Pigmentatry nevi on face have unique patterns and implications: the concept of blaschko` sline for pigmentatry line by Nilendusarma from Indian journal of dermatology http:www.e-ijd.org 2017;57.

5. Clinical mycological evaluation of dermatophytosis at tertiary care hospital in central in diaby Shyamgovind from International journal of research in dermatology 2018aug: 4(3);409-414.

6. Diagnostic procedures in dermatology by 
Prof HF Jordan department of dermatology ; university of Stellenbosch (http://acade mic.sun.ac.za/stellmed /articles /dermatology /an10909.htm(2006).

7. Cutaneous hypopigmentatry disorders by Kalegowdadeepadarshan from WWW.Odermatol.com

8. Prevalence of different species of Malassezia causing Pityriasis versicolor and sites of distribution of lesions in a tertiary care hospital in Kolkata ,India by Sampurna Biswas pramanik from International journal of current Microbiology and applied science volume 4 number (2015) ppt, 471-478.

9. Krishnan A, Thapa DM. Mophological and pigmentary variation tineaversi color in south Indian patients. Indian J Dematol 2003; 48:83-6.

10. Vijaya D, Nagarathnamma T, Anand
Kumar BH, Rajesh R, Satish N, Savitha $\mathrm{G}$, et al., Study of Pityriasisversicolor. The Antiseptic 1998; 95:133.

11. Aljebre SH, Alzayir AA, Abdulghani M, Osman OO. Pigmentary changes of tineaversicolor in dark-skinned patients. Int J Dermatol 2001; 40:273-5.

12. Tarazooie B, Kordbacheh P, Zaini F, Zomorodian K, Zeraati H, et al.Study of distribution of Malassezia species in patients with Pityriasis versicolor and healthy individuals iTehran, Iran. BMC Dermatol 2004; 4:1-6 .

13. Sundar Khadka et.al, (2016) Clinicomycological Characterization of Superficial Mycoses from a Tertiary Care Hospital in Nepal. Dermatology Research and Practice, Volume 2016, Article ID 9509705, 7 pages.

\section{How to cite this article:}

Rizwanasheik, J. Manonmoney and Leela, K. V. 2020. An Observational Study on Infectious Etiology of Hypo and Hyper Pigmented Macular Lesions in the Skin in a Tertiary Care Hospital. Int.J.Curr.Microbiol.App.Sci. 9(08): 1112-1121. doi: https://doi.org/10.20546/ijcmas.2020.908.122 